Bull. Austral. Math. Soc.

VOL. 42 (1990) [483-486]

\title{
HOMOTHETIC MAPS OF DISTINGUISHING SPACE-TIMES
}

\author{
Jin-HWAN KIM
}

We show that if $(M, g)$ is a distinguishing space-time, then any distance homothetic map from $(M, g)$ onto arbitrary space-time $\left(M^{\prime}, g^{\prime}\right)$ is a homothetic map; in particular, every distance preserving map is an isometry.

\section{INTRODUCTION AND PRELIMINARIES}

Homothetic maps preserve the metric tensors up to a constant factor and LeviCivita connections, also causal structures in the Lorentzian case. It is well known that in the positive definite case every distance preserving map of one Riemannian manifold onto another is an isometry and vice-versa. In the Lorentzian case, this result is not valid. Beem [1] has shown that for strongly causal space-times, any distance homothetic map is a homothetic map in the usual sense of metric tensors. In this paper, we obtain such a result for distinguishing space-times. We know well that distinguishingness is weaker condition than strong causality.

By a space-time we mean a time-oriented Lorentzian manifold $(M, g)$. If there is a future directed timelike (respectively, nonspacelike) curve from $p$ to $q$, we write $p \ll q$ (respectively, $p<q$ ). $p \leqslant q$ means that $p<q$ or $p=q$. The chronological future $I^{+}(p)$ and the causal future $J^{+}(p)$ of $p$ are defined as follows.

$$
I^{+}(p)=\{q \in M \mid p \ll q\}, J^{+}(p)=\{q \in M \mid p \leqslant q\}
$$

Dually, the chronological past $I^{-}(p)$ and the causal past $J^{-}(p)$ of $p$ are defined.

Given two points $p, q \in M$, the Lorentzian distance $d(p, q)$ is defined as the supremum of lengths of nonspacelike curves from $p$ to $q$ whenever $q \in J^{+}(p)$. If $q \notin J^{+}(p)$, then $d(p, q)=0$. Unlike the Riemannian distance function, the Lorentzian distance function is not necessarily finite-valued, nor even continuous. Also, this function is not a true metric.

A space-time $(M, g)$ is future (respectively, past) distinguishing if $I^{+}(p)=I^{+}(q)$ (respectively, $I^{-}(p)=I^{-}(q)$ ) implies $p=q$; equivalently, for any $p \in M$ and any neighbourhood $U_{p}$ of $p$, there exists a neighbourhood $V_{p} \subset U_{p}$ of $p$ such that no future (respectively, past) directed nonspacelike curve through $p$ which leaves $V_{p}$ ever returns to it. $(M, g)$ is distinguishing if it is future and past distinguishing.

Received 9 January 1990

Copyright Clearance Centre, Inc. Serial-fee code: 0004-9729/90 \$A2.00+0.00. 


\section{MaIN RESUlt}

Throughout this section, we let $(M, g)$ and $\left(M^{\prime}, g^{\prime}\right)$ be space-times and let $d$ and $d^{\prime}$ denote the Lorentzian distance functions of $(M, g)$ and $\left(M^{\prime}, g^{\prime}\right)$ respectively.

A map $f$ from $(M, g)$ onto $\left(M^{\prime}, g^{\prime}\right)$ is said to be distance homothetic if there exists a constant $c>0$ such that $d^{\prime}(f(p), f(q))=c d(p, q)$ for all $p, q \in M$. If $c=1$, then $f$ is said to be distance preserving. If a map from $(M, g)$ onto $\left(M^{\prime}, g^{\prime}\right)$ satisfies $p \ll q$ if and only if $f(p) \ll f(q)$ for all $p, q \in M$, then $f$ will be called a chronal map. A one-to-one chronal map from $(M, g)$ onto $\left(M^{\prime}, g^{\prime}\right)$ is said to be a chronal isomorphism.

We first prove several lemmas which are used in obtaining the main theorem.

LEMMA 1. If a map $f:(M, g) \rightarrow\left(M^{\prime}, g^{\prime}\right)$ is a distance homothetic map, then $f$ is a chronal map.

ProOF: Since $d^{\prime}(f(p), f(q))=c d(p, q), p \ll q$ if and only if $d(p, q)>0$ if and only if $d^{\prime}(f(p), f(q))>0$ if and only if $f(p) \ll f(q)$.

LEMMA 2. Let $(M, g)$ be a future (respectively, past) distinguishing space-time and $\left(M^{\prime}, g^{\prime}\right)$ be an arbitrary space-time. If $f$ is a chronal map from $(M, g)$ onto $\left(M^{\prime}, g^{\prime}\right)$, then

(a) $f$ is one-to-one and hence it is a chronal isomorphism.

(b) $\left(M^{\prime}, g^{\prime}\right)$ is future (respectively, past) distinguishing.

Proof: (a) Suppose $f(p)=f(q)$. Then

$\begin{array}{llcl}x \in I^{+}(p) & \text { if and only if } & p \ll x & \text { if and only if } \\ f(p) \ll f(x) & \text { if and only if } & f(x) \in I^{+}(f(p))=I^{+}(f(q)) & \text { if and only if } \\ f(q) \ll f(x) & \text { if and only if } & q \ll x & \text { if and only if } \\ x \in I^{+}(q) . & & & \end{array}$

Thus $I^{+}(p)=I^{+}(q)$. Since $(M, g)$ is future distinguishing, $p=q$. Hence $f$ is one-to-one and also a chronal isomorphism.

(b) Since $p \ll q$ if and only if $f(p) \ll f(q)$ for all $p, q \in M, f\left(I^{+}(p)\right)=I^{+}(f(p))$ for all $p \in M$. Now suppose $I^{+}(z)=I^{+}(w)$ with $z=f(p)$ and $w=f(q)$. Then $f\left(I^{+}(p)\right)=I^{+}(f(p))=I^{+}(f(q))=f\left(I^{+}(q)\right)$. By $(\mathrm{a}), f$ is one-to-one and therefore $I^{+}(p)=I^{+}(q)$. Since $(M, g)$ is future distinguishing, $p=q$. So $z=w$. Hence $\left(M^{\prime}, g^{\prime}\right)$ is future distinguishing. Similarly for the past part.

This lemma is not valid for arbitrary space-times (even if $(M, g)$ is chronological). Let $M=S^{1} \times R$ be the space-time with the Lorentzian metric $d s^{2}=d \theta d t$ and let $x_{0}$ be a fixed point of $S^{1}$. Define $f: M \rightarrow M$ by $f(x, t)=\left(x_{0}, t\right)$ for all $(x, t) \in M$. Then $f$ is a chronal map but it is not one-to-one. 
Using the above Lemma 2 and Theorem 2 in Malament [3] (or Theorem 4 of Vyas and Akolia [4]), we have the following.

LEMмA 3. Let $(M, g)$ be a distinguishing space-time and $\left(M^{\prime}, g^{\prime}\right)$ be an arbitrary space-time. Let $f$ be a chronal map from $(M, g)$ onto $\left(M^{\prime}, g^{\prime}\right)$ (not assumed to be either continuous or one-to-one). Then $f$ is a conformal diffeomorphism.

Now we prove the main theorem.

THEOREM 4. Let $(M, g)$ be a distinguishing space-time and $\left(M^{\prime}, g^{\prime}\right)$ be an arbitrary space-time. If $f$ is a distance homothetic map of $(M, g)$ onto $\left(M^{\prime}, g^{\prime}\right)$, then $f$ is a homothetic map. That is, $f$ is a diffeomorphism and there is a constant $k>0$ such that $f$ maps $g$ onto $\mathbf{k g}^{\prime}$. In particular, every map of a distinguishing space-time $(M, g)$ onto itself which preserves Lorentzian distance function is an isometry.

Proof: By the above lemmas, $\left(M^{\prime}, g^{\prime}\right)$ is distinguishing and $f$ is a conformal diffeomorphism. For a point $p \in M$, let $\langle V \subset U\rangle_{p}$ mean that $U$ is a convex normal neighbourhood of $p$ and $V$ is a small neighbourhood $V$ of $p$ contained in $U$ such that whenever $q \in V$ with $p \ll q$, the unique future-directed timelike geodesic segment $\overline{p q}$ from $p$ to $q$ in $U$ lies in $V$ and the length of $\overline{p q}$ is equal to $d(p, q)$, and if $q, r \in V$ with $p \ll r \ll q$, then the reverse triangle inequality $d(p, q) \geqslant d(p, r)+d(r, q)$ is valid with strict equality if and only if $r$ is on the geodesic segment $\overline{p q}$. In distinguishing space-time, any point $p$ has $\langle V \subset U\rangle_{p}$. Now for any $p \in M$, choose $\left\langle U_{1} \subset U\right\rangle_{p}$ and $\left\langle U_{2} \subset U\right\rangle_{p}$, and $\left\langle W_{1} \subset W\right\rangle_{f(p)}$ such that $f(U) \subset W, f\left(U_{1}\right) \subset W_{1}$ and $f^{-1}\left(W_{1}\right) \subset U_{2}$. Let $q \in U_{1}$ with $p \ll q$. Since $f$ is distance homothetic, $f$ maps the timelike geodesic segment $\overline{p q}$ in $U$ to the timelike geodesic segment $\overline{f(p) f(q)}$ joining $f(p)$ to $f(q)$ in $W$ and the lengths of $\overline{p q}$ and $\overline{f(p) f(q)}$ are given by $d(p, q)$ and $d^{\prime}(f(p), f(q))$ respectively. It follows that $f$ maps $g$ onto the tensor $c^{-2} g^{\prime}$. This completes the proof.

We remark that when the hypothesis of distinguishingness in Theorem 4 is weakened to future or past distinguishingness, distance homothetic maps are not necessarily homothetic maps (see Example of Beem [1, p.798]).

\section{REFERENCES}

[1] J.K. Beem, 'Homothetic maps of the space-time distance function and differentiability', Gen. Relativity Gravitation 9 (1978), 793-799.

[2] J.K. Beem and P.E. Ehrlich, Global Lorentzian Geometry (Marcel Dekker, 1981).

[3] D.B. Malament, 'The class of continuous timelike curves determine the topology of space-times', J. Math. Phys. 18 (1977), 1399-1404.

[4] U.D. Vyas and G.M. Akolia, 'Chronal isomorphism', Gen. Relativity Gravitation 16 (1984),

$1045-1051$. 
Department of Mathematica

College of Science

Yeungnam University

P.O. Box 713-749, Kyungsan

Korea 